\title{
FACTORES QUE INFLUYEN EN EL USO DEL HÁBITAT POR EL VENADO COLA BLANCA (ODOCOILEUS VIRGINIANUS COUESI) EN LA SIERRA DEL LAUREL, AGUASCALIENTES, MÉXICO
}

\author{
Salvador Martín Medina TORRES, Edmundo GARCíA MOYA, \\ Marcelo Márquez Olivas, Humberto VAquera Huerta, \\ Angélica Romero Manzanares, Mario MArtínez MeneS \\ Programa orientado en Botánica, Campus Montecillo, Colegio de Postgraduados. Monte- \\ cillo Estado de México. Carretera México-Texcoco, km 36.5. C.P. 56230. \\ E-mail: smedinat@colpos.mx, edmundo@colpos.mx, molivas@colpos.mx, \\ hvaquera@colpos.mx, dahly@colpos.mx,mmario@colpos.mx.
}

\section{RESUMEN}

Durante la época seca de 2006 se registró el aprovechamiento del hábitat por el venado cola blanca (Odocoileus virginianus couesi) en la Sierra del Laurel de Aguascalientes. El objetivo de este estudio fue analizar, a través de regresión logística, algunos atributos biofísicos del hábitat que influyen en la probabilidad de que algunos sitios sean utilizados, en mayor o menor proporción, por el venado. El tipo de vegetación, la pendiente, la altitud y la distancia al agua influyeron de manera significativa $(\mathrm{p}<0.01)$ en la probabilidad de aprovechamiento del hábitat por el venado. Los umbrales de estas últimas tres variables se determinaron para una probabilidad de aprovechamiento alta $p(y \geq 0.5)$ y variaron en función del tipo de vegetación. En términos generales, las mayores probabilidades de aprovechamiento se obtuvieron en el matorral subtropical. Para una probabilidad de aprovechamiento alta, este tipo de vegetación tuvo una densidad de 50 árboles de Ipomoea murucoides Roem \& Schult y 350 arbustos de Eysenhardtia polystachya Ortega Sarg. por hectárea; así como una altura promedio del estrato arbustivo de $2.0 \mathrm{~m}$. En virtud de que el matorral subtropical constituye el hábitat más escaso y amenazado por actividades agrícolas y ganaderas en la Sierra del Laurel, y con base en las evidencias aportadas por este estudio, es necesario promover acciones entre los dueños de la tierra para realizar una mejor gestión de este tipo de vegetación, que es de gran importancia para el venado en la región.

Palabras clave: Venado cola blanca, Odocoileus virginianus couesi, hábitat, matorral subtropical, Sierra del Laurel, Aguascalientes, México.

\footnotetext{
ABSTRACT

Habitat use by the Coues white tailed deer (Odocoileus virginianus couesi) was documented during the dry season of 2006, in the Sierra del Laurel, Aguascalientes, Mexico. Based on logistic regression analyses, the objective of this study was to determine which floristic-structural and topographical features influence the probability of habitat use by the Coues deer. Vegetation, slope, altitude and distance to water were significant $(\mathrm{p}<0.01)$ in the probability of habitat use. The thresholds for slope, altitude and distance were estimated for a high probability of use $p(y \geq 0.5)$ and varied due to vegetation type. Sites on subtropical vegetation with 50 trees of Ipomoea murucoides Roem. \& Schult by hectare and 350 shrubs of Eysenhardtia polystachya Ortega Sarg., and mean height of the shrub layer
} 
over the 2.0 meters, had a probability of use greater than 50 percent. However this vegetation type is scarce and threatened by agricultural development in the Sierra del Laurel. The results of this research clearly point out the need to promote management and conservation strategies of this type of vegetation since it is the preferred habitat of the Coues deer, which in turn should be considered as an asset by the state holders and an option to derive additional income from hunting fees and other economic activities pertaining to a multiple use of the land.

Key words: Coues deer, Odocoileus virginianus couesi, habitat, subtropical shrub, Sierra del Laurel, Aguascalientes, Mexico.

\section{INTRODUCCIÓN}

El venado cola blanca (Odocoileus virginianus Zimmermann 1780) es uno de los herbívoros silvestres que se encuentra en casi todo el territorio nacional, con excepción de la península de Baja California, sobre una gran variedad de ecosistemas que van desde las regiones secas del norte, pasando por los bosques templados, hasta las regiones tropicales del sur (Leopold 1985, Galindo-Leal y Weber 1998). Su adaptación, tamaño, amplia distribución, calidad de su carne para el consumo humano y la estética de sus astas, han hecho del venado cola blanca una de las especies cinegéticas más importantes de Norteamérica y la más manejable en forma intensiva y extensiva en México (Galindo-Leal y Weber 1998, Villarreal 1999).

De las 14 subespecies de venado cola blanca en México, Odocoileus virginianus couesi (Coues y Yarrow 1875) es una de las de mayor interés cinegético y la que ocupa mayor territorio (Villarreal 1999, Galindo-Leal y Weber 1998). Su distribución abarca desde Arizona, en los Estados Unidos de Norteamérica, hasta Jalisco en México, a lo largo de la Sierra Madre Occidental (Ezcurra y Gallina 1981), e incluye Aguascalientes (Galindo-Leal y Weber 1998). En Aguascalientes, como en otras partes, ha sido utilizado como un recurso alimentario para complementar la dieta de los habitantes de las comunidades rurales, de manera particular los de la sierra. Asimismo, constituye un recurso alimentario para diversos depredadores, dispersa semillas y ejerce un efecto importante en la estructura de la vegetación de la Sierra Madre Occidental (Galindo-Leal y Weber 1998).

El venado cola blanca, en Aguascalientes, estuvo al borde de la extinción en la década de los 70 del siglo pasado debido, principalmente, a la cacería no regulada y a las alteraciones y disminución de su hábitat (Medina 1986, Romo 1987). Para revertir esa tendencia y promover la investigación de la especie, en 1975 se creó el "Programa de Conservación y Aprovechamiento Cinegético del Venado Cola Blanca en la Sierra Fría de Aguascalientes" y como resultado de este programa, a finales de 1990 sus poblaciones ya se habían recuperado (Villalobos 1998). De manera desafortunada, las acciones de protección del venado cola blanca no se han promovido con el mismo entusiasmo en las otras áreas del estado, como en la Sierra del Laurel, donde la caza furtiva prevalece, y tampoco se ha motivado a los dueños de la tierra para que realicen una gestión apropiada de este recurso y diversifiquen sus actividades productivas. 
La cacería legal del venado cola blanca, en Aguascalientes, se ha practicado desde los años 70 del siglo pasado bajo la supervisión del gobierno federal y sin la participación de los dueños de la tierra, hasta mediados de los años 80 (Medina 1986). Sin embargo, es importante señalar que la cacería de subsistencia ya se realizaba con anterioridad. En 1996 se propuso en México una forma novedosa de gestión de este recurso, a través de las Unidades de Manejo para la Conservación de la Vida Silvestre (UMA), en las que los dueños de la tierra podían obtener un beneficio económico por el aprovechamiento cinegético de la fauna que manejaran en sus predios. En 1998, por lo menos, cinco predios particulares de Aguascalientes empezaron a ejercer los primeros permisos de aprovechamiento cinegético del venado cola blanca bajo este nuevo enfoque (Kobelkowsky 2000). En la actualidad, el número de UMA y de venados cazados se ha incrementado en la entidad y podría verse amenazado de nuevo, ya que algunos propietarios de las UMA prefieren introducir especies exóticas en sus predios, lo que conlleva al uso indiscriminado de cercos para el aseguramiento y retención de esos semovientes; barreras que limitan el libre movimiento de las poblaciones de venado y de otras especies nativas.

Los criterios en las UMA para decidir y autorizar la caza de un número determinado de venados se sustentan, principalmente, en estudios poblacionales más que en información sobre el uso (considerado en este trabajo como aprovechamiento) y selección del hábitat o, en el mejor de los casos, se consideran índices de calidad del hábitat basados en información de otros entornos de Norteamérica. Es razonable pensar que cualquier población de venados, en una región determinada, hará un aprovechamiento selectivo de su hábitat y que tal selección dependerá de ciertos atributos como la topografía, la disponibilidad de agua, la estructura de la vegetación, los factores inherentes a la gestión, e incluso, los cambios en la disponibilidad de los recursos, lo que en última instancia se traduciría en diferencias importantes en cuanto a su abundancia y, por consiguiente, en su potencial de aprovechamiento. Sin embargo, estudios recientes (Wayne 2001, Moore et al. 2003, Morrison et al. 2003, Félix et al. 2007) han demostrado que es posible extraer información valiosa y útil que permita identificar aquellas características o atributos del hábitat que influyan sobre la selección de sitios por la especie, mediante la utilización de métodos de regresión logística (Manly et al. 1993). Estos métodos pueden utilizarse bajo situaciones en donde las variables de predicción no se distribuyen de manera normal y en las que algunas o todas sean discretas o categóricas (Johnson 2000); además de que sus resultados son una función de probabilidad cuya escala de valores ( 0.0 a 1.0) es análoga a un índice de calidad del hábitat. Ejemplos como los de Moore et al. (2003) y Marshal (2006) con venado bura; Mysterud e Ims (1998) en faisán de collar y Wayne (2001), Morrison et al. (2003) y Félix et al. (2007) con venado cola blanca, demuestran la pertinencia de este método. 
El propósito de este estudio fue analizar, mediante regresión logística, atributos del hábitat que influyen en la probabilidad de que los sitios sean utilizados, en mayor o menor proporción, por el venado cola blanca en la Sierra del Laurel.

\section{MATERIAL Y MÉTODOS}

Área de estudio. La Sierra del Laurel se localiza al sur-occidente del estado de Aguascalientes, dentro del municipio de Calvillo, entre los $21^{\circ} 42^{\prime}$ y $21^{\circ} 51^{\prime}$ de latitud Norte, y entre los $102^{\circ} 47^{\prime}$ y $102^{\circ} 35^{\prime}$ de longitud Oeste. Tiene una superficie de 14,609.87 ha y forma parte de la Unidad de Manejo Forestal Regional (UMAFOR) de la Asociación Sierra del Laurel, A.C. (Figura 1).

El área de estudio se localiza en la subprovincia fisiográfica Sierras y Valles Zacatecanos. Tiene una topografía accidentada, con altitudes que varían de 1,700 a $2,760 \mathrm{~m}$. Geológicamente data de la era cenozoica, del período terciario, y esta compuesta por rocas ígneas extrusivas. Los suelos dominantes en las partes altas corresponden al tipo litosol, caracterizados por tener una profundidad menor a los 10 $\mathrm{cm}$, limitado por rocas y tepetate, mientras que en las partes bajas predominan los feozem háplico, que se caracterizan por presentar una capa superficial obscura, suave y rica en materia orgánica y nutrimentos, de fertilidad moderada (INEGI 2005).

La Sierra del Laurel constituye uno de los principales parteaguas que drenan hacia el valle de Huajucar, con origen en las partes altas de la sierra propiamente dicha, y que drenan hacia el sistema de los Ríos Gil-Malpaso-Calvillo, que forman parte de la sub-cuenca del Río Juchipila, perteneciente a la región hidrológica 12 (MedinaTorres 2006).

En la Sierra del Laurel existen tres tipos de clima de acuerdo con la clasificación de Koppen modificada por García (García-CONABIO 1988); el templado subhúmedo con lluvias en verano $\mathrm{C}(\mathrm{w})$ en las partes altas de la Sierra; semicálido semiseco $\left(\mathrm{BS}_{1} \mathrm{~h}\right)$ en las partes bajas del valle de Huajucar y el cálido semi-seco $\left(\mathrm{BS}_{1} \mathrm{k}\right)$ en la zona oriental, hacia el valle de Aguascalientes (INEGI 2005). La precipitación promedio anual es de $550 \mathrm{~mm}$, de acuerdo al promedio histórico documentado entre 1949 y 1998 (Medina-Torres 2006).

La vegetación del área de estudio se puede describir en tres grandes grupos: bosque de encino, matorral subtropical y pastizal inducido.

El bosque de encino (Quercus spp) se caracteriza por la presencia de árboles de 6 a 12 m de altura. Puede ser abierto o muy denso (Huber y Ritna 2003) y con algunas áreas mezcladas con pino y estratos arbustivos de densidad variable de Arctostaphylus spp. y Arbutus spp, con diversos grados de disturbio. Se localiza entre los 2,000 y 2,740 m de altitud y en algunos sitios se advierte perturbación por aprovechamientos forestales e incendios, que han trasformado la vegetación original en manchones densos de Mimosa monancistra Benth. y M. aculeaticarpa Ort. 

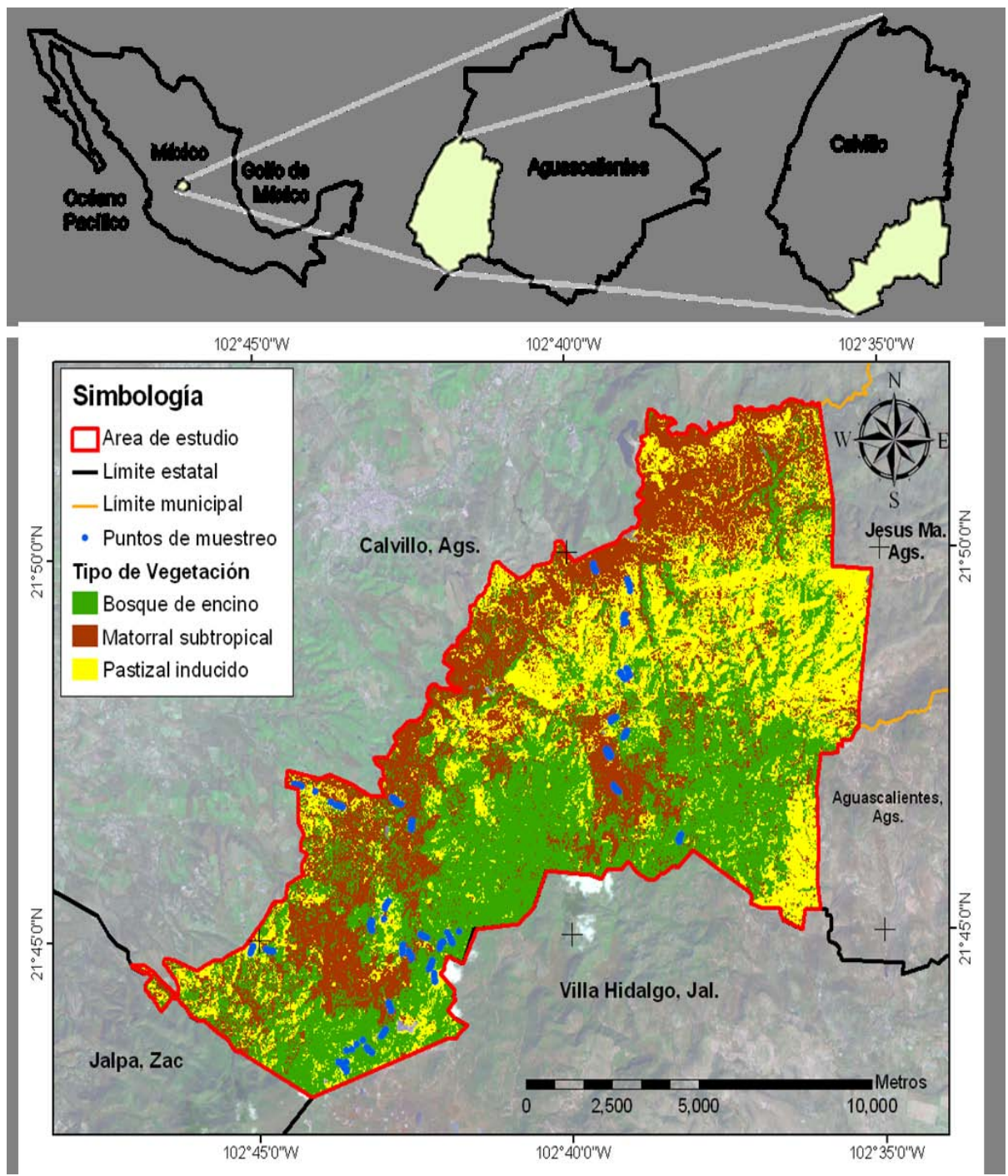

Figura 1. Ubicación del área de estudio en la Sierra del Laurel, Municipio de Calvillo, Aguascalientes.

El matorral subtropical lo integran las comunidades vegetales formadas por arbustos o árboles bajos, inermes o espinosos, que incluye grupos heterogéneos de asociaciones vegetales dominadas por especies indicadoras de disturbio, o francamente propias de asociaciones secundarias y se localiza entre los 1,500 y 2,000 m de altitud (Rzedowski y McVaugh 1966). La mayor parte de las especies que lo 
constituyen pierden su follaje durante un período prolongado del año. Los principales componentes son: Ipomoea murucoides Roem. et Schult. Bursera roseana Rzed., Eysenhardtia polystachya Ortega Sarg., Acacia pennatula, (Schlecht. \& Cham.) Benth., Forestiera spp, Erythrina spp., y Opuntia spp, entre otras (Huber y Ritna 2003, Flores-Olvera y Lindig-Cisneros 2005). Esta comunidad vegetal ha sido afectada por el desmonte para el establecimiento de huertas de guayaba, cítricos, agave azul y maíz, expansión urbana y el aprovechamiento de especies vegetales nativas (Siqueiros-Delgado et al. 2006).

El pastizal inducido corresponde a las comunidades de gramíneas y herbáceas que surgen cuando es eliminada la vegetación natural como consecuencia de tala, desmontes o incendios (Huber y Ritna 2003). El sobrepastoreo en estas áreas ha ocasionado un predominio de arbustivas que corresponden a etapas seriales iniciales, entre las cuales destacan Dodonaea viscosa (L.) Jacq, Mimosa spp y Acacia spp y, en menor proporción, de algunas especies de la comunidad vegetal original (bosques de encino, matorral, etc.).

Método. El área de muestreo se determinó mediante la cartografía digital que generó el Instituto del Medio Ambiente del Estado de Aguascalientes (IMAE) y mapas topográficos, escala 1:50,000. Previo al estudio, se visitaron 56 sitios con el propósito de identificar y caracterizar los tipos de vegetación y compararlos con la cartografía disponible. Cada sitio fue georeferenciado con un navegador Mobil-Maper Pro ${ }^{\circledR}$, con un error de ubicación menor de $3 \mathrm{~m}$. Con los puntos obtenidos se generó un archivo de capas vectoriales en el programa Arc-View 3.2 de ESRI ${ }^{\circledR}$, que después fue utilizado en un procedimiento de clasificación supervisada de una imagen satelital, LANDSAT 7 ETM 1999 path / row 29/45 del 15 de agosto de 1999, previamente corregida por sus efectos atmosféricos y topográficos, con el clasificador duro de Fisher del programa Idrisi Kilimanjaro ${ }^{\circledR}$. Los sitios resultantes fueron reclasificados en los tres tipos de vegetación descritos con anterioridad.

Para el análisis del aprovechamiento de hábitat se utilizaron los registros de presencia / ausencia de grupos fecales de venado depositados durante 120 días por período de muestreo (febrero-abril y mayo-julio), obtenidos de un estudio poblacional de esta especie, simultáneo a esta investigación. Los datos se recabaron de 174 puntos de muestreo de un total de 180, los cuales fueron repartidos entre 30 transectos asignados proporcionalmente a cada tipo de vegetación, ya que al perderse seis puntos de muestreo pertenecientes a dos de los transectos durante el seguimiento de mayo-julio, no fue posible obtener información de ellos.

En el análisis de los datos en el estudio poblacional mencionado, se demostró que no hubo un efecto significativo del período de muestreo sobre la densidad de grupos fecales por parcela, por lo que cada parcela se consideró utilizada (aprovechamiento de hábitat $=1$ ) si se registró la presencia de grupos fecales de venado, al menos durante una de las dos visitas realizadas. En cada transecto de $200 \mathrm{~m}$ de longitud se 
ubicaron seis puntos distribuidos de manera sistemática cada $40 \mathrm{~m}$ a partir de su inicio. Cada punto representó el centro de una parcela circular de $9.3 \mathrm{~m}^{2}$, sobre la que se registró la presencia o ausencia de grupos fecales de venado. Cada punto fue georeferenciado y caracterizado de acuerdo con sus variables topográficas y disponibilidad de agua. Las variables topográficas a considerar fueron: altitud (m), pendiente (\%) y azimut (grados), derivadas de un modelo digital de elevación con una resolución de $10 \mathrm{~m}$, a partir de la capa vectorial de curvas de nivel escala 1:50,000 generadas por el INEGI, con el programa Idrisi Kilimanjaro ${ }^{\circledR}$. La disponibilidad de agua se evaluó en términos de la distancia de cada punto al cuerpo de agua más cercano, para lo cual se utilizó un mapa vectorial de los cuerpos de agua que se localizaron en la zona, seleccionando sólo aquellos que tuvieran agua durante el período de muestreo. Para cada punto, se determinó esta distancia a partir del Sistema de Información Geográfica. Los hallazgos de excretas de venado encontrados fuera de las parcelas, o entre transectos, también fueron registrados y georeferenciados. A estos registros se les añadió la información del tipo de vegetación, topografía y disponibilidad de agua, con lo cual se obtuvo una base de 306 registros de aprovechamiento de hábitat en total; 174 registros de excretas dentro de las parcelas de muestreo y 132 fuera de las parcelas.

Debido a limitaciones en la accesibilidad a los sitios y la disponibilidad de recursos y tiempo, en 101 de los 174 puntos y procurando evaluar al menos tres dentro de cada transecto, se realizó con un distanciómetro Hagglof DME ${ }^{\circledR}$ el

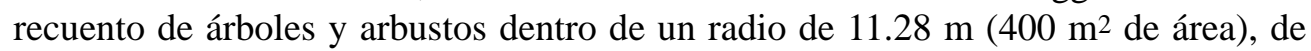
acuerdo con una variante a la técnica empleada en el inventario nacional forestal (SEMARNAT-CONAFOR 2004). La vegetación fue clasificada como arbórea sí su diámetro a nivel de pecho era superior a los $7.5 \mathrm{~cm}$ (SEMARNAT-CONAFOR 2004), o su altura igual o mayor a $3 \mathrm{~m}$, o como arbustos sí su altura era inferior a $3 \mathrm{~m}$ y de tallos leñosos y ramificados (Durán-García 1995). Cada individuo dentro de la parcela fue contado e identificado. En el caso de los árboles, el área basal total y por especie fue estimada con el método de Bitterlich (Dieguez-Aranda et al. 2003), con un dendrómetro Cruz-All ${ }^{\circledR}$ y un factor de área basal (FAB) de 10 pies cuadrados por acre $\left(2.30 \mathrm{~m}^{2} / \mathrm{ha}\right)$, mientras que para los arbustos se empleó un dendrómetro de madera construido conforme al procedimiento descrito por Hays et al. (1981), con un FAB de $50 \mathrm{~m} 2 /$ ha. El porcentaje de sombreado se obtuvo con un esferodensiómetro cóncavo de Forestry ${ }^{\circledR}$ a $60 \mathrm{~cm}$ sobre el suelo en el punto central de la parcela. La altura promedio de los árboles (altura máxima + mínima / 2) se estimó con un altímetro Haga ${ }^{\circledR}$ y la de los arbustos con una cinta métrica metálica graduada en $\mathrm{cm}$. La densidad de Quercus eduardii, I. murucoides, Bursera roseana y E. polystachya, se obtuvo para verificar sí estas variables influyen en el aprovechamiento del hábitat por el venado, ya que los lugareños asocian su presencia con la abundancia de tales especies de plantas. 
Para evaluar el aprovechamiento del hábitat por el venado cola blanca en la Sierra del Laurel, se formularon dos preguntas fundamentales como hipótesis de trabajo:

a) ¿La probabilidad de que un sitio cualquiera sea utilizado por el venado cola blanca, en la Sierra del Laurel, depende del tipo de vegetación, de la distancia al agua y de al menos un atributo de la topografía?

b) ¿La probabilidad de aprovechamiento del hábitat por el venado cola blanca podrá explicarse en función de al menos uno de los atributos florístico-estructurales de la vegetación utilizada?

Para responder a la primera interrogante y conocer la distribución espacial de la probabilidad de aprovechamiento del hábitat por el venado cola blanca en la Sierra del Laurel, mediante un mapa digital, se utilizó un análisis de regresión logística (Manly et al. 1993) con un modelo de selección ascendente (Stepwise), mediante el SAS ${ }^{\circledR}$ (2007), utilizando como variable de respuesta la presencia o ausencia de grupos fecales de venado (aprovechamiento de hábitat) y como variables predecibles, el tipo de vegetación, la pendiente, el azimut, la altitud, y la distancia al agua.

El mapa de probabilidad de aprovechamiento del hábitat se generó con el calculador de imágenes (Image Calculator) del programa Idrisi Kilimanjaro ${ }^{\circledR}$, en el cual se introdujeron los parámetros obtenidos en el modelo de regresión logística, y las imágenes raster del tipo de vegetación, distancia al agua y las variables topográficas, en un procedimiento de cálculo basado en la Ecuación (1) de regresión logística (Manly et al. 1993):

$$
p(y=1) x=\frac{\exp \left(\beta_{0}+\beta_{1} x_{1}+\ldots+\beta_{p} x_{p}\right)}{1+\exp \left(\beta_{0}+\beta_{1} x_{1}+\ldots+\beta_{p} x_{p}\right)}
$$

donde $p(y=1)$ es la probabilidad de que un determinado sitio sea utilizado por el venado, en función de las variables de predicción $x$ ya descritas.

Con un proceso de reclasificación (instrucción RECLASS, Idrisi Kilimanjaro ${ }^{\circledR}$ ), se generó una zonificación del área de estudio en dos clases de probabilidad de uso: alta $p(y \succeq 0.5)$ y baja 1-p $(y \succeq 0.5)$. En seguida, se realizó una tabulación cruzada entre el mapa de vegetación con el mapa de clases de probabilidad de aprovechamiento (instrucción CROSTAB, Idrisi Kilimanjaro ${ }^{\circledR}$ ), para obtener un mapa con los tres tipos de vegetación que presentaron una probabilidad de aprovechamiento alta $p(y \geq 0.5)$.

Por último, se obtuvo la media $(\mu)$ y la desviación estándar $(\sigma)$ de las variables que resultaron significativas en el modelo del primer análisis, con probabilidad de aprovechamiento alta (instrucción EXTRACT, programa Idrisi Kilimanjaro ${ }^{\circledR}$ ), lo que permitió describir cada tipo de vegetación con base en su topografía y su disponibilidad de agua.

Considerando que entre las variables del medio físico sólo la distancia al agua es susceptible de ser intervenida, se modeló su comportamiento en interacción con las 
variables topográficas que resultaron significativas en el modelo logístico, lo que permitió determinar la distancia al agua mínima requerida, para una probabilidad alta de aprovechamiento por el venado.

Con la finalidad de probar la segunda hipótesis de trabajo, se realizó un segundo análisis de regresión logística con un modelo de selección ascendente (Stepwise), sobre las 101 parcelas en las que se evaluó la vegetación, utilizando la misma variable de respuesta (presencia o ausencia de grupos fecales de venado) y como variables de predicción, el tipo de vegetación del sitio evaluado, y sus atributos florístico-estructurales.

Mediante los estadísticos básicos de las variables significativas por tipo de vegetación en el modelo anterior, se construyeron escenarios para modelar su comportamiento, y determinar los valores necesarios para una alta probabilidad de aprovechamiento por el venado cola blanca.

\section{RESULTADOS}

El primer modelo logístico demostró que la probabilidad de que un sitio dado sea aprovechado por el venado cola blanca, en la Sierra del Laurel, depende del tipo de vegetación, de la distancia al agua, de la pendiente y de la altitud $(-2 \log \mathrm{L}=$ $366.65, \mathrm{~g} 1=5, \mathrm{p}<0.0001$ ). Los parámetros del modelo se presentan en el cuadro 1 y con sus valores se derivó la Ecuación 2.

$\left.p(y=1) x=\frac{\exp \left(-6.0422-0.0222\left(x_{1}\right)+0.0032\left(x_{2}\right)-0.0007\left(x_{3}\right)-0.7869\left(x_{4}\right)+1.3844\left(x_{5}\right)\right.}{1+\exp \left(-6.0422-0.0222\left(x_{1}\right)+0.0032\left(x_{2}\right)-0.0007\left(x_{3}\right)-0.7869\left(x_{4}\right)+1.3844\left(x_{5}\right)\right.} 2\right)$

donde $p(y=1)=$ es la probabilidad de que un determinado sitio sea utilizado por el venado, $x_{1}=$ pendiente $(\%), x_{2}=$ altitud, $x_{3}=$ distancia al agua $(\mathrm{m}), x_{4}=$ bosque de encino y $x_{5}=$ matorral subtropical.

Cuadro 1. Parámetros del modelo de las variables del medio físico

\begin{tabular}{lcccc}
\hline \multicolumn{1}{c}{ Variable } & Parámetro & Error estándar & $\begin{array}{c}\text { Chi-cuadrada } \\
\text { de Wald }\end{array}$ & Pr > Chi² \\
\hline Intersección & -6.042 & 2.131 & 8.040 & 0.005 \\
Pendiente (\%) & -0.022 & 0.010 & 4.772 & 0.029 \\
Altitud (m) & 0.003 & 0.001 & 10.337 & 0.001 \\
Distancia al agua (m) & -0.001 & 0.000 & 4.366 & 0.037 \\
Bosque de encino & -0.787 & 0.258 & 9.305 & 0.002 \\
Matorral subtropical & 1.384 & 0.222 & 38.922 & 0.000 \\
\hline
\end{tabular}


El mapa de probabilidad de aprovechamiento del hábitat en el área de estudio generado con la ecuación (2) se muestra en la figura 2 y la zonificación de la categoría de aprovechamiento del hábitat con probabilidad alta se presenta en la figura 3. El $69.6 \%\left(101.4 \mathrm{~km}^{2}\right)$ del área de estudio tuvo una probabilidad de aprovechamiento baja 1-p $(y \searrow 0.5)$, mientras que el $30.4 \%\left(44.3 \mathrm{~km}^{2}\right)$ presentó una probabilidad de aprovechamiento alta $p(y \geq 0.5)$

El bosque de encino, con alta probabilidad de ser aprovechado por el venado cola blanca, abarcó una superficie de 433.25 ha (7.38\% del total disponible) y se caracterizó por presentar una pendiente del $17 \pm 11 \%$, una altitud de $2457 \pm 79 \mathrm{~m}$, y una distancia al agua de $564 \pm 304 \mathrm{~m}$. Los sitios del matorral subtropical con mayor probabilidad de aprovechamiento tienen una extensión de 3,328.00 ha (73.26\% del total disponible) y presentaron una pendiente del $28 \pm 17 \%$, una altitud de $2053 \pm 184 \mathrm{~m}$, y una distancia al agua de $846 \pm 508 \mathrm{~m}$. Finalmente, el pastizal inducido con mayor probabilidad de aprovechamiento abarca una

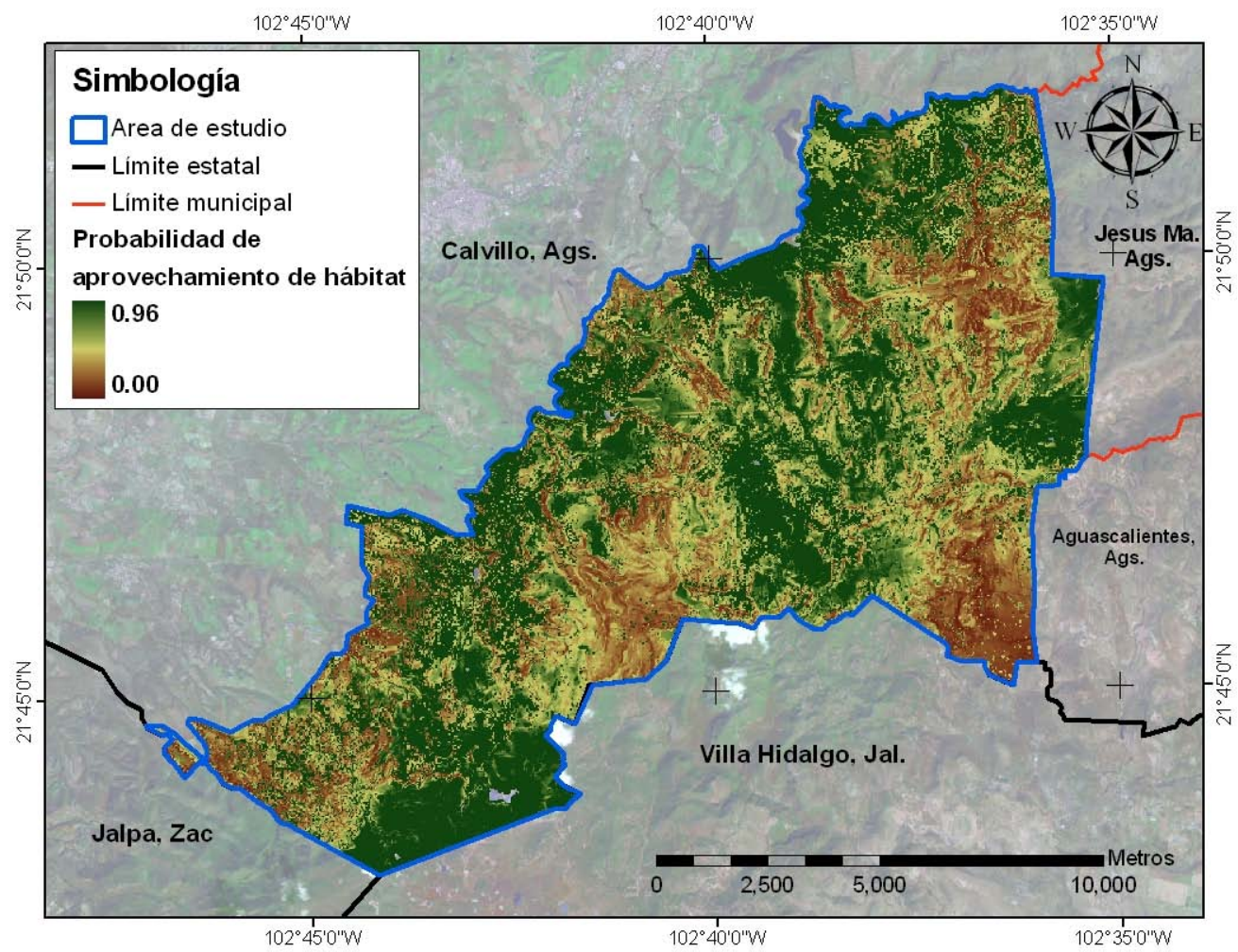

Figura 2. Mapa de probabilidad de aprovechamiento del hábitat por el venado cola blanca en la Sierra del Laurel de Aguascalientes, con base en el tipo de vegetación, la altitud (m), la pendiente (\%) y distancia al agua $(\mathrm{m})$. 


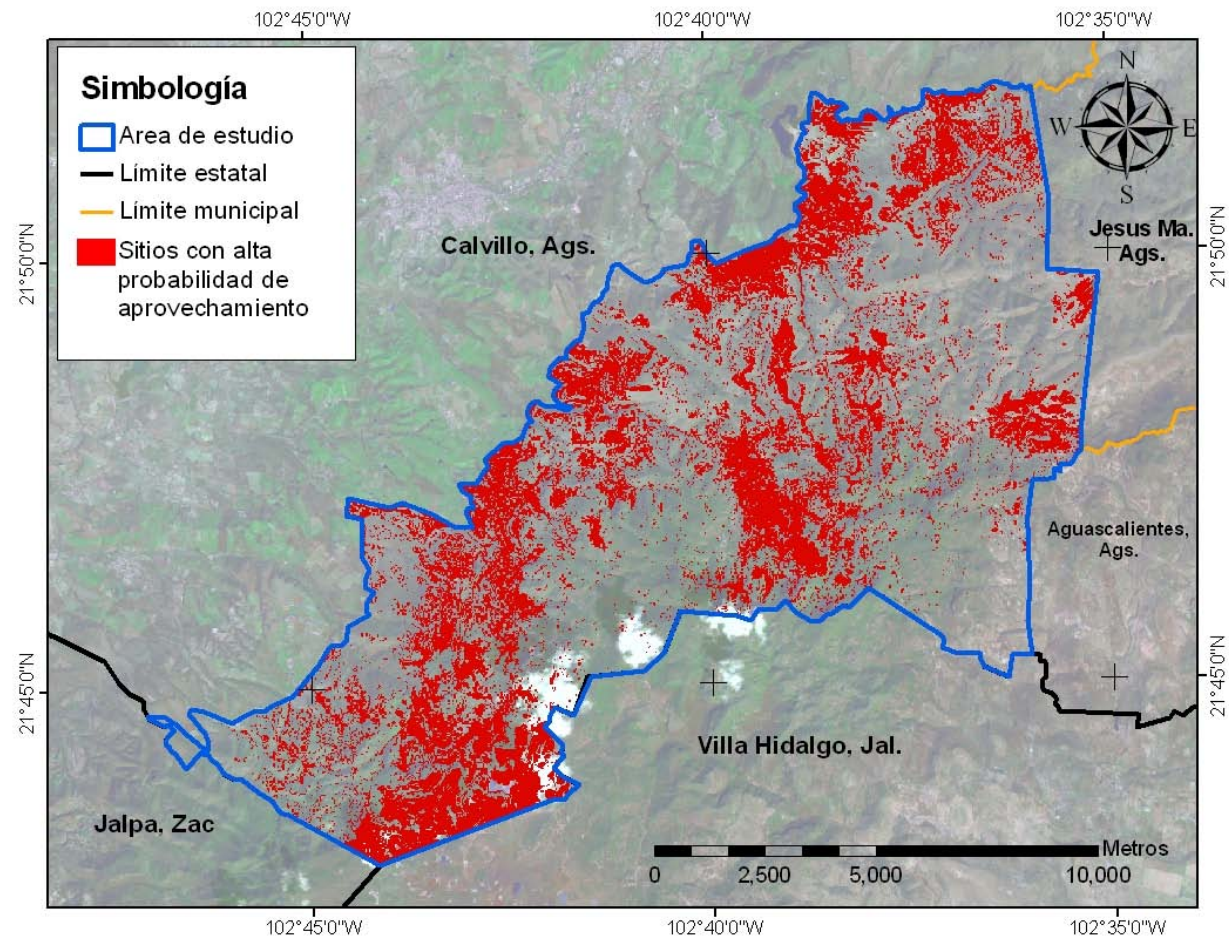

Figura. 3. Mapa de sitios con alta probabilidad de aprovechamiento del hábitat por el venado cola blanca en la Sierra del Laurel de Aguascalientes.

extensión de 672.40 ha $(16.16 \%$ del total disponible) y se caracterizó por presentar una pendiente de $16 \pm 12 \%$, una altitud de $2239 \pm 145 \mathrm{~m}$, y una distancia al agua de $537 \pm 429 \mathrm{~m}$ (Cuadro 2)

El modelado de la distancia al agua demostró que los valores mínimos requeridos para una alta probabilidad de aprovechamiento por el venado cola blanca, en el área de estudio, variaron en función de la vegetación, la pendiente y altitud del hábitat. En los sitios con alta probabilidad de aprovechamiento por el venado, la distancia al agua se torna crítica cuando se alcanza la mayor pendiente y la mínima altitud, comportamiento que varió en función del tipo de vegetación utilizado. El comportamiento de las variables en el modelo demostró que la distancia al agua requerida para una alta probabilidad de aprovechamiento del hábitat, se incrementa conforme la pendiente disminuye.

En el bosque de encino, la distancia al agua no debe ser mayor de $230 \mathrm{~m}$ en pendientes superiores al $28 \%$ y altitudes mínimas de 2,378 m. En contraste, en el matorral subtropical se observó una mayor tolerancia a distancias mayores al agua, ya que la distancia mínima para una probabilidad alta de aprovechamiento fue de $480 \mathrm{~m}$ en 
Cuadro 2. Promedio $(\mu)$, desviación estándar $(\sigma)$ y extremos de la pendiente, altitud y distancia al agua $(\mu \pm \sigma$, para una probabilidad de uso de hábitat alta.

\begin{tabular}{|c|c|c|c|c|}
\hline \multicolumn{5}{|c|}{ Pendiente (\%) } \\
\hline Tipo de vegetación & $\mu$ & ( & $\mu-\sigma$ & $\mu+c$ \\
\hline Bosque de encino & 17 & 11 & 7 & 28 \\
\hline Matorral subtropical & 28 & 17 & 10 & 45 \\
\hline Pastizal inducido & 16 & 12 & 5 & 28 \\
\hline \multicolumn{5}{|c|}{ Altitud (msnm) } \\
\hline Tipo de vegetación & $\|$ & ( $T$ & $u-0$ & $11+0$ \\
\hline Bosque de encino & 2,457 & 79 & 2,378 & 2,535 \\
\hline Matorral subtropical & 2,053 & 184 & 1,869 & 2,237 \\
\hline Pastizal inducido & 2,239 & 145 & 2,094 & 2,384 \\
\hline \multicolumn{5}{|c|}{ Distancia al Agua $(\mathrm{m})$} \\
\hline Tipo de vegetación & $\mu$ & ( & $\mu-(T$ & $\mu+c$ \\
\hline Bosque de encino & 564 & 304 & 260 & 868 \\
\hline Matorral subtropical & 846 & 508 & 338 & 1,354 \\
\hline Pastizal inducido & 537 & 429 & 108 & 966 \\
\hline
\end{tabular}

terrenos abruptos (pendientes no mayores al 45\%). El pastizal inducido fue el que mostró ser el hábitat mas vulnerable a la disponibilidad de agua, ya que bajo condiciones extremas de máxima pendiente y mínima altitud ( $28 \%$ y 2,094 m respectivamente) la distancia al agua mínima para una probabilidad de aprovechamiento alta fue de $50 \mathrm{~m}$. Un resumen de de estos valores se presenta en el cuadro 3.

En el análisis de regresión logística, aplicado a los atributos florístico-estructurales del hábitat, sobre las 101 parcelas de muestreo en las que se evaluó la vegetación, se obtuvo un modelo donde la altura media del estrato arbustivo, la densidad de Ipomoea murucoides y de Eysenhardtia polystachya fueron significativas $(-2 \log \mathrm{L}=90.14$, $\mathrm{g} \mathrm{l}=3, \mathrm{p}<0.0001$ ). Los parámetros de este modelo se presentan en el cuadro 4 . Con estos parámetros se construyó el modelo logístico que permitió el modelado de sus respectivas variables, representado por la ecuación 3: 
Cuadro 3. Valores mínimos requeridos de la distancia al agua por tipo de vegetación para una probabilidad de aprovechamiento alta, bajo tres escenarios distintos de pendiente y altitud.

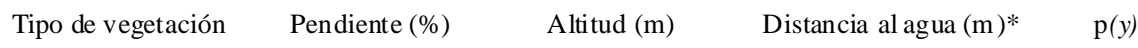

\begin{tabular}{|c|c|c|c|c|c|c|c|}
\hline \multirow{3}{*}{ Matorral subtropical } & $11-0$ & 10 & $1+a$ & 2,237 & $1+c$ & 1,354 & 0.80 \\
\hline & $!$ & 28 & $!$ & 2,053 & $1 d+c$ & 1,354 & 0.60 \\
\hline & $1+a$ & 45 & $u-0$ & 1,869 & $U$ & 480 & 0.50 \\
\hline \multirow{3}{*}{ Bosque de encino } & $11-0$ & 7 & 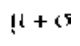 & 2,535 & $11+c$ & 868 & 0.63 \\
\hline & !l & 17 & !l & 2,457 & $1 d+c$ & 868 & 0.52 \\
\hline & $1+a$ & 28 & $u-0$ & 2,378 & $U$ & 230 & 0.50 \\
\hline \multirow{3}{*}{ Pastizal inducido } & $1-0$ & 5 & $1+a$ & 2,384 & $11+c$ & 966 & 0.70 \\
\hline & !1 & 17 & !l & 2,239 & $1+a$ & 966 & 0.53 \\
\hline & $11+6$ & 28 & $u-0$ & 2,094 & $U$ & 50 & 0.50 \\
\hline
\end{tabular}

Nota: $*$ = variable modelada. $\mu=$ media, $\sigma=$ desviación estándar, $U=$ umbral para una probabilidad $\geq$ $0.50, \mathrm{p}(\mathrm{y})=$ probabilidad de aprovechamiento de hábitat

$$
p(y=1) x=\frac{\exp \left(-3.2381-0.0167\left(x_{1}\right)+0.0025\left(x_{2}\right)+0.7794\left(x_{3}\right)\right.}{1+\exp \left(-3.2381-0.0167\left(x_{1}\right)+0.0025\left(x_{2}\right)+0.7794\left(x_{3}\right)\right.}
$$

donde $p(y=1)=$ es la probabilidad de que un determinado sitio sea utilizado por el venado, $x_{1}=$ árboles de $I$. murucoides por ha, $x_{2}=$ arbustos de E. polystachya por ha, y $x_{3}=$ altura media de arbustos (m).

El bosque de encino presentó una baja densidad de I. murucoides por hectárea $(2.40 \pm 3.96)$. En este tipo de vegetación se registró la mayor altura del estrato arbustivo $(2.45 \pm 0.41 \mathrm{~m})$, donde estuvo ausente $E$. polystachya. En contraste, el matorral subtropical presentó los valores mas altos de densidad de I. murucoides y $E$. polystachya por hectárea $(45.59 \pm 23.08$ y $319.85 \pm 144.05)$ respectivamente, y una altura de $2.05 \pm 0.39 \mathrm{~m}$ en el estrato arbustivo. El pastizal natural presentó una densidad de $16.67 \pm 26.51$ árboles de $I$. murucoides por hectárea, y una altura media de arbustos de $1.38 \pm 0.18 \mathrm{~m}$. E. polystachya no estuvo presente en este tipo de vegetación (Cuadro 5). 
Medina et al. Factores que influyen en el uso del habitat del venado cola blanca

Cuadro 4. Parámetros del modelo de las variables del medio biótico

\begin{tabular}{lcccc}
\hline \multicolumn{1}{c}{ Variables } & Parámetro & Error estándar & $\begin{array}{c}\text { Chi-cuadrada } \\
\text { de Wald }\end{array}$ & Pr > Chi \\
\hline Intersección & -3.2381 & 0.6372 & 25.8207 & $<0.0001$ \\
Ipomoea murucoides / ha & 0.0167 & 0.0061 & 7.4457 & 0.0064 \\
Eysenhardtia polystachya / ha & 0.0025 & 0.0011 & 4.7996 & 0.0285 \\
Altura media de arbustos (m) & 0.7794 & 0.2234 & 12.1667 & 0.0005 \\
\hline
\end{tabular}

Cuadro 5. Estadísticos de las variables que fueron significativas en el modelo logístico aplicado a los atributos florístico-estructurales de la vegetación

\begin{tabular}{|c|c|c|c|c|c|}
\hline \multicolumn{6}{|c|}{ Ipomoea murucoides por hectárea } \\
\hline Tipo de vegetación & $\mathrm{n}$ & $\mathrm{m}$ & es & Min & Max \\
\hline Bosque de encino & 52 & 2.40 & 13.84 & 0.00 & 100.00 \\
\hline Matorral subtropical & 34 & 45.59 & 73.71 & 0.00 & 275.00 \\
\hline Pastizal inducido & 15 & 16.67 & 45.18 & 0.00 & 175.00 \\
\hline \multicolumn{6}{|c|}{ Eysenhardtia polystachya / ha } \\
\hline Tipo de vegetación & $\mathrm{n}$ & $\mathrm{m}$ & es & Min & Max \\
\hline Bosque de encino & 52 & 0.00 & 0.00 & 0.00 & 0.00 \\
\hline Matorral subtropical & 34 & 319.85 & 70.80 & 0.00 & $1,400.00$ \\
\hline Pastizal inducido & 15 & 0.00 & 0.00 & 0.00 & 0.00 \\
\hline \multicolumn{6}{|c|}{ Altura media de arbustos $(\mathrm{m})$} \\
\hline Tipo de vegetación & $\mathrm{n}$ & $\mathrm{m}$ & es & Min & $\operatorname{Max}$ \\
\hline Bosque de encino & 52 & 2.45 & 0.20 & 0.00 & 7.00 \\
\hline Matorral subtropical & 34 & 2.05 & 0.19 & 0.00 & 5.90 \\
\hline Pastizal inducido & 15 & 1.38 & 0.09 & 1.05 & 2.25 \\
\hline
\end{tabular}

Nota: $\mathrm{n}=$ tamaño de muestra, $\mathrm{m}=$ media, es = error estandar, Min = valor mínimo, Max = valor máximo. 
La probabilidad del aprovechamiento de hábitat por el venado cola blanca estuvo en función de la interacción de las tres variables anteriores y su modelado demostró que una alta probabilidad de aprovechamiento $p(y \geq 0.5)$ se asoció a sitios de matorral subtropical, con un mínimo de 50 árboles de $I$. murucoides y 350 arbustos de $E$. polystachya por hectárea, así como una altura media del estrato arbustivo no inferior a $2 \mathrm{~m}$. En el bosque de encino, solamente en sitios con vegetación secundaria arbustiva cuya altura media fuera menor a $4.2 \mathrm{~m}$, la probabilidad de aprovechamiento por el venado fue alta $p(y \searrow .5)$. En contraste, en sitios de pastizal inducido con un mínimo de 100 árboles de I. murucoides por hectárea y una altura media del estrato arbustivo mayor de $2 \mathrm{~m}$ es posible obtener una probabilidad de aprovechamiento superior a 0.5 (Cuadro 6).

Cuadro 6. Modelado de la densidad por hectárea de Ipomoea murucoides, Eysenhardtia polystachya y la altura media de arbustos por tipo de vegetación, para determinar los umbrales requeridos para una probabilidad alta de aprovechamiento de hábitat por el venado cola blanca

\begin{tabular}{|c|c|c|c|c|c|c|c|}
\hline \multirow[t]{2}{*}{$\begin{array}{c}\text { Tipo de } \\
\text { Vegetación }\end{array}$} & \multicolumn{2}{|c|}{ I. murucoides / ha } & \multicolumn{2}{|c|}{ E. polystachya / ha } & \multicolumn{2}{|c|}{$\begin{array}{l}\text { Altura media } \\
\text { arbustos (m) }\end{array}$} & \multirow{2}{*}{$\begin{array}{c}\text { Probabilidad } \\
\text { de uso de } \\
\text { hábitat } \\
0.19\end{array}$} \\
\hline & $m-e s$ & 0.43 & $m-e s$ & 0.00 & $m-e s$ & 2.25 & \\
\hline \multirow{4}{*}{$\begin{array}{l}\text { Bosque de } \\
\text { encino }\end{array}$} & $m$ & 2.40 & $m$ & 0.00 & $m$ & 2.45 & 0.22 \\
\hline & $m+e s$ & 4.38 & $m+e s$ & 0.00 & $m+e s$ & 2.66 & 0.25 \\
\hline & $\operatorname{Max}$ & 100.00 & $\operatorname{Max}$ & 0.00 & $\operatorname{Max}$ & 7.00 & 0.98 \\
\hline & Min & 0.00 & $m$ & 0.00 & $V U$ & 4.20 & 0.51 \\
\hline \multirow{5}{*}{$\begin{array}{l}\text { Matorral } \\
\text { subtropical }\end{array}$} & $m-e s$ & 34.25 & $m-e s$ & 249.05 & $m-e s$ & 1.86 & 0.35 \\
\hline & $m$ & 45.59 & $m$ & 319.85 & $m$ & 2.05 & 0.48 \\
\hline & $m+e s$ & 56.93 & $m+e s$ & 390.65 & $m+e s$ & 2.25 & 0.61 \\
\hline & $\operatorname{Max}$ & 275.00 & $\operatorname{Max}$ & 1400.00 & $\operatorname{Max}$ & 5.90 & 1.00 \\
\hline & $U$ & 50.00 & $U$ & 350.00 & $U$ & 2.00 & 0.51 \\
\hline \multirow{5}{*}{$\begin{array}{l}\text { Pastizal } \\
\text { inducido }\end{array}$} & $m-e s$ & 5.32 & $m-e s$ & 0.00 & $m-e s$ & 1.29 & 0.10 \\
\hline & $m$ & 16.67 & $m$ & 0.00 & $m$ & 1.38 & 0.13 \\
\hline & $m+e s$ & 28.01 & $m+e s$ & 0.00 & $m+e s$ & 1.47 & 0.16 \\
\hline & $\operatorname{Max}$ & 175.00 & $\operatorname{Max}$ & 0.00 & $\operatorname{Max}$ & 2.25 & 0.81 \\
\hline & $U$ & 100.00 & $m$ & 0.00 & $U$ & 2.02 & 0.50 \\
\hline
\end{tabular}

Nota: $\mathrm{m}=$ media, es $=$ error estándar, Max = valor máximo, Min = valor mínimo, $\mathrm{U}=$ umbral

\section{DISCUSIÓN}

El primer modelo logístico sugiere que la probabilidad de que un determinado sitio sea aprovechado por el venado está en función del tipo de vegetación, pendiente, altitud y distancia al agua. En los dos modelos logísticos generados, se encontró que las probabilidades de aprovechamiento más altas se presentaron en el matorral 
subtropical; lo que sugiere que el tipo de vegetación es uno de los atributos que más influyen en la selección del hábitat por el venado cola blanca, tal y como lo documentó Boulanger et al. (2000).

A diferencia de lo encontrado por Galindo-Leal y Weber (1998), la alta probabilidad asociada al matorral subtropical revela que el venado en la Sierra del Laurel prefirió este tipo de vegetación sobre el bosque de encino, cuando este último ha sido el hábitat preferido en la Sierra de la Michilía, Durango, de acuerdo con los autores citados. La selección por vegetación caducifolia ha sido documentada por otros autores (Wayne, 2001, Mandujano et al. 2004), y puede ser debida a la gran diversidad de especies que ofrecen mayor cobertura y variedad de alimento a los venados. Depew (1976) encontró que el venado cola blanca tiende a ser más abundante en áreas con mayor proporción de vegetación arbustiva, lo que puede atribuirse a una preferencia por sitios con vegetación secundaria, más que sitios con vegetación madura o "clímax" (Olson 1992).

Al considerar la pendiente, los resultados del primer modelo demostraron la importancia de los rasgos topográficos y su influencia sobre la selección del hábitat por el venado cola blanca, ya que ésta se asocia con pendientes inferiores a 30\%, coincidiendo con las tendencias que otros autores han documentado en Norteamérica (Boulanger et al. 2000, Wayne 2001) y América Central (Segura 1998). Otro rasgo topográfico que influye en que determinados sitios sean seleccionados por el venado es la altitud (Boulanger et al. 2000). En este estudio se encontró que la probabilidad de aprovechamiento del hábitat aumenta a medida que la altitud del terreno se incrementa.

La distancia al agua influyó en la selección del hábitat por el venado cola blanca en la Sierra del Laurel. Se encontró que los sitios con una probabilidad de aprovechamiento alta tuvieron una distancia promedio al agua entre 537 y $846 \mathrm{~m}$ entre los tres tipos de vegetación evaluados. Estos valores fueron mayores que los encontrados por Bello y Equihua (2001) y Ortiz-Martínez et al. (2005), aunque están dentro de los valores recomendados para la gestión del venado cola blanca, los cuales oscilan entre 700 y 800 m (Villarreal 1999, SDR 2007). Las sequías frecuentes y la falta de corrientes de agua permanente en la Sierra del Laurel (Medina-Torres 2006), obligan al venado a depender del rocío, del agua contenida en algunas plantas y frutos, y de las pocas represas que llegan a conservar agua durante todo el año. Es de llamar la atención que en el matorral subtropical la probabilidad de aprovechamiento por el venado cola blanca se asocia a distancias al agua más grandes que en los otros tipos de vegetación, lo que sugiere que en ese entorno encuentra otras fuentes para satisfacer sus necesidades de agua, tal y como otros autores lo han documentado en hábitats caducifolios semejantes al matorral subtropical del área de estudio (Mandujano et al. 2004, Villarreal Espino-Barros y Marín-Fuentes 2005).

En el segundo modelo se comprobó que la probabilidad de aprovechamiento es mayor en el matorral subtropical que en los otros dos tipos de vegetación analizados. 
Las variables florístico-estructurales que favorecieron dicho aprovechamiento son la altura media de los arbustos, y la densidad de Ipomoea murucoides y Eysenhardtia polystachya por hectárea. Probabilidades altas de aprovechamiento, asociadas a una cierta altura media del estrato arbustivo, pueden ser debidas a la satisfacción de los requerimientos del venado en términos de cobertura, tanto térmica como de protección (Galindo-Leal y Weber 1998, Ortiz-Martínez et al. 2005). En el matorral subtropical la altura media de los arbustos podría ser un factor importante como refugio bajo condiciones climáticas extremas, ya que el clima irregular, con sequías recurrentes y prolongadas de la Sierra del Laurel (Medina-Torres 2006), incrementa los requerimientos de cobertura térmica bajo estas condiciones (Bello y Equihua 2001). Gallina et al. (2005) documentaron que durante la época de gestación (marzo a junio), el venado cola blanca tiene menor actividad. Esta condición puede deberse a la necesidad de factores termo-reguladores que le protejan de temperaturas elevadas, para lo cual son necesarios hábitats particularmente densos. Uresk et al. (1999) encontraron que las hembras de venado cola blanca seleccionaron sitios con una altura media de la vegetación de $1.01 \mathrm{~m}$, mientras que esta altura en sitios elegidos al azar fue de $0.75 \mathrm{~m}$. Christopher et al. (2002) encontraron que la selección de hábitats específicos por el venado cola blanca se explica mejor por la cobertura vegetal en el estrato arbustivo, ya que éste proporciona cobertura térmica, de escape y alimento. Estos investigadores mencionan, además, que los sitios con vegetación arbustiva se aprovechan de 1.5 a 4.7 veces más en relación con su disponibilidad, en tanto que los sitios con sotobosque escaso o inexistente fueron evitados. Otro beneficio que ofrece la vegetación arbustiva es que afecta la visibilidad vertical y protege al venado contra el hombre y los depredadores (Galindo-Leal y Weber 1998, Ortiz-Martínez et al. 2005). Un comportamiento semejante fue documentado por Kilgo et al. (1998) en poblaciones de venado cola blanca que durante la temporada de caza evitaron áreas taladas, o hábitats abiertos, y seleccionaron sitios con vegetación madura y cobertura arbustiva densa. En el área de estudio, la caza furtiva del venado puede ser un factor adicional que influya en la selección del matorral subtropical.

La importancia de Eysenhardtia polystachya como fuente de alimento para el venado cola blanca ha sido documentada por varios autores (Villalobos 1998, Villarreal 1999, Villarreal Espino-Barros et al. 2007). En la Mixteca Poblana, Villarreal EspinoBarros et al. (2007) encontró que, entre las especies ramoneadas por el venado cola blanca, las leguminosas constituyen el $20 \%$. Dentro de esta familia, E. polystachya es una especie básica en la composición botánica de la dieta y está disponible entre los meses de mayo a octubre. Esta especie es particularmente abundante y característica del matorral subtropical en la Sierra del Laurel, y se deduce que debe ser importante como proveedora de alimento y cobertura ya que los lugareños reconocen que es una especie preferida por el venado cola blanca (Medina 1986, Villalobos 1998). A pesar 
de ser considerada como una especie de alto valor forrajero, es frecuente que ésta sea afectada por ganaderos locales en su intento por controlar los crecimientos secundarios de gatuño (Mimosa monancistra y M. aculeaticarpa) en sus agostaderos, situación que ocasiona una disminución en la calidad del hábitat para el venado cola blanca.

Otro de los atributos florístico-estructurales que contribuyeron a explicar la selección del hábitat por el venado cola blanca en la Sierra del Laurel fue la densidad de árboles Ipomoea murucoides por hectárea, Esta especie ha sido documentada como una planta proveedora de agua para el venado cola blanca, ya que contiene de manera aproximada $76.25 \%$ de agua (Villarreal Espino-Barros y Marín-Fuentes 2005). Esta característica puede ser importante en el matorral subtropical de la Sierra del Laurel, donde hay una baja disponibilidad de agua. Debido a la alta densidad de I. murucoides en el matorral subtropical, es posible que la distancia al agua para altas probabilidades de aprovechamiento sea mayor con respecto a los otros tipos de vegetación.

Amenazas y factores de presión hacia el hábitat. Siqueiros-Delgado et al. (2006) mencionan que el matorral subtropical de la Sierra del Laurel es uno de los tipos de vegetación más afectados por el desarrollo agropecuario, pues ha visto mermada su extensión por cambios de uso de la tierra tales como el establecimiento de huertas de guayaba y, más recientemente, para el cultivo del agave azul. La ganadería es otra de las actividades que también afectan de manera negativa a este tipo de vegetación. Con mucha frecuencia, los ganaderos de la región realizan prácticas de control de ese matorral y afectan la calidad del hábitat del venado cola blanca en la Sierra del Laurel. La erradicación de I. murucoides con herbicidas, con el propósito de evitar sus efectos tóxicos sobre el ganado bovino es muy común en la zona. Se han realizado estudios en los que se considera a I. murucoides como una especie tóxica para el ganado y por eso se procede a su erradicación (GonzálezAlaniz 1997). Durante octubre de 2006, la Procuraduría Estatal de Protección al Ambiente del estado de Aguascalientes documentó un caso en el municipio de Calvillo, en donde se estimó que en más de 100 ha se había eliminado hasta el 83\% de los árboles de I. murucoides, así como a todos los arbustos de esta misma especie.

En la actualidad, el uso principal de la tierra en el área de estudio es el pecuario, consistente en sistemas de producción vaca-becerro, de apacentamiento continuo todo el año (Medina-Torres 2006). También es frecuente que gran parte de las tierras sean rentadas para apacentar ganado, ya que sus propietarios se encuentran trabajando en los Estados Unidos de Norteamérica, lo que fomenta el descuido, el aprovechamiento excesivo de los recursos naturales y la proliferación de prácticas de gestión inadecuadas (Observación personal). Otras actividades como el desmonte selectivo del matorral subtropical para abrir espacios a la agricultura constituyen una amenaza seria para el venado cola blanca, ya que con esto se afecta la estructura vegetal en términos de altura del estrato arbustivo, que junto con la densidad de $I$. 
murucoides y E. polystachya, son las variables que mejor explicaron la selección del hábitat por la especie.

Una forma de inducir a revalorar el matorral subtropical por parte de los ganaderos y contribuir a una diversificación productiva, es la gestión sustentable de otros recursos naturales (SEMARNAP 1997), por ahora no considerados en la práctica pecuaria tradicional. Uno de los recursos es el aprovechamiento del venado cola blanca nativo de la zona. Si continúan las prácticas de erradicación de $I$. murucoides, en el matorral subtropical de la Sierra del Laurel por parte de los ganaderos, resulta evidente que habría una disminución importante en la probabilidad de aprovechamiento del hábitat por el venado, lo cual a su vez sería causa de que se pierda la oportunidad para su conservación y su eventual aprovechamiento sostenido.

En cuanto al método desarrollado en este trabajo, es importante considerar que las variables de uso común en los estudios dasométricos convencionales que se utilizan para la elaboración de los programas de gestión forestal, pueden ser utilizadas exitosamente para evaluar el aprovechamiento del hábitat por el venado u otra especie silvestre de interés. Solo es necesario introducir, en la rutina de muestreo forestal, el registro de indicios y observaciones que denoten la presencia o ausencia de la especie a estudiar.

Las variables del medio físico que más influyeron en el aprovechamiento del hábitat por el venado cola blanca en la Sierra del Laurel son: tipo de vegetación, pendiente, altitud y disponibilidad de agua. Esta última, en términos de distancia a su fuente o reservorio más cercano, constituye un factor crítico en la probabilidad de aprovechamiento del hábitat por el venado, tanto en el pastizal inducido como en el bosque de encino, ya que dicha distancia no deberá ser mayor a 50 y $230 \mathrm{~m}$ en pendientes del $28 \%$, respectivamente. La distancia mínima al agua podrá incrementarse conforme la pendiente disminuya, de tal forma que en pendientes del 10 al 5\%, la distancia deberá ser de 200 a 370 m en el pastizal inducido, y de 450 a 600 en el bosque de encino. Por el contrario, en el matorral subtropical el agua no es un factor limitativo.

Es altamente probable que el venado utilice más el matorral subtropical que los otros tipos de vegetación por la densidad de Ipomoea murucoides y Eysenhardtia polystachya por hectárea, y por la altura media del estrato arbustivo. Las características bióticas que debe reunir un sitio determinado para una alta probabilidad de aprovechamiento son, para el matorral subtropical: altura media del estrato arbustivo de $2.0 \mathrm{~m}, 50$ árboles de $I$. murucoides por ha, y 350 arbustos de E. polystachya por ha; en el bosque de encino: estratos arbustivos cuya altura media no sea inferior a $4.2 \mathrm{~m}$; y en el pastizal inducido, una densidad mínima de 100 árboles de I. murucoides por hectárea, y una altura media de arbustos por encima de $2 \mathrm{~m}$. 
Agradecimientos. El primer autor agradece al Colegio de Postgraduados, al CONACYT, a la Asociación Sierra del Laurel, A.C., a la Presidencia Municipal de Calvillo, a la Procuraduría Estatal de Protección al Ambiente (PROESPA) y al Instituto del Medio Ambiente del Estado de Aguascalientes (IMAE), por las facilidades otorgadas para la realización de esta investigación. Un especial agradecimiento a la Biol. Gabriela Delgadillo Quezada, por su colaboración y apoyo en la realización de los trabajos de campo. Este estudio se dedica a la grata memoria de los guardabosques de la PROESPA y entrañables amigos del primer autor: Miriam Limón Díaz de León (Aura), Jaime Hernández Esquivel (Ganso), Carlos Alberto Muñoz Molina (Chaman) y Gustavo Eduardo Valdez Romo (Panda), caídos en el cumplimiento de su deber el 24 de junio de 2007.

\section{LITERATURA CITADA}

Bello, J. S. G. \& M. Equihua. 2001. Characterization and habitat preferences by white-tailed deer in Mexico. Journal of Range Management. 54:537-545.

Boulanger, J. G., K G. Poole, J. Gwilliam, G. P. Woods, J. Krebs, \& I. Parfitt. 2000. Winter Habitat Selection by White-Tailed Deer in The Pend D'oreille Valley, Southeastern British Columbia. Columbia Basin Fish \& Wildlife Compensation Program. British Columbia. 48 p.

Christopher, S. D, J. A. Jenks, S. L. Griffin, L. A. Rice, \& K. F. Higgins. 2002. White-tailed deer habitats in the central Black Hills. Journal of Range Management. 55:242-252.

Depew, J. J. 1976- (August 2004). Habitat selection and movement patterns of cattle and white-tailed deer in a temperate savanna. Master's thesis, Texas A\&M University. In: http://handle.tamu.edu/1969.1/2616.

Dieguez-Aranda, U., M. Barrio, \& F. Castedo. 2003. Dendrometría. Editorial Mundiprensa. Madrid. $327 \mathrm{p}$.

Durán-García, R. 1995. Diversidad Florística de los Petenes en Campeche. Acta Botánica Mexicana 31:73-84.

Ezcurra, E. \& S. Gallina, 1981. Biology and population dynamics of white-tailed deer in northwestern Mexico. Pp 77-106. In: Ffolliott, P. F. \& Gallina S. (eds.) Deer Bioloy, Habitat Requirements, and Management in Western North America. Instituto de Ecología, México.

Felix, A. B., D. P. Walsh, B. D. Hughey, H. Campa III, \& S. R. Winterstein. 2007. Applying Landscape-Scale Habitat-Potential Models to Understand Deer Spatial Structure and Movement Patterns. Journal of Wildlife Management. 71(3):804-810.

Flores-Olvera, M. H. \& R. Lindig-Cisneros. 2005. La Lista de nombres vulgares y botánicos de árboles y arbustos propicios para repoblar los bosques de la República de Fernando Altamirano y José Ramírez a más de 110 años de su publicación. Revista Mexicana de Biodiversidad 76: 11- 35.

Galindo-Leal, C. \& M. Weber. 1998. El Venado de la Sierra Madre Occidental. Ecología, Manejo y Conservación. EDICUSA-CONABIO. México, D. F. 272 p.

Gallina, S., P. Corona-Zárate, \& J. Bello. 2005. El Comportamiento del Venado Cola Blanca en Zonas Semiáridas del Noroeste de México. Pp.193-204. En: Sánchez-Cordero V. \& Medellín R. A. (Eds.) Contribuciones Mastozoológicas en Homenaje a Bernardo Villa. Instituto de Biología, Instituto de Ecología, UNAM; CONABIO. México.

García, E. - Comisión Nacional para el Conocimiento y Uso de la Biodiversidad (CONABIO), 1998. Climas (Clasificación de Koppen, modificado por García). Escala 1:1 000 000. México.

González-Alaniz, M. H. 1997. Evaluación de los agostaderos de Aguascalientes y su potencial de mejoramiento. Cuadernos de Trabajo Agricultura y Recursos Naturales. No. 65. Gobierno del Estado Aguascalientes, México. 44 p.

Hays, R. L., C. Summers \& W. Seitz. 1981. Estimating Wildlife habitat Variables. Biological Services Program. Fish and Wildlife Service. U.S. Department of the Interior. Washington, D. C. 113 p. 
Huber O. \& R. Ritna. 2003. Glosario Fitoecológico de las Américas. Vol. 2. México, América Central e Islas del Caribe: países hispanoparlantes. Ediciones UNESCO-Coro Lab Humboldt. UNESCO, París, Francia. 474 p.

INEGI. 2005. Anuario Estadístico de Aguascalientes, edición 2005. En: http://www.inegi.gob.mx/est/ contenidos/espanol/sistemas/aee05/estatal/ags/index.htm.

Johnson, D. E. 2000. Métodos Multivariados Aplicados al Análisis de Datos. Internacional Thomson Editores, S.A. de C.V. México, D.F. 566 p.

Kilgo, J. C., R. F. Labisky, \& D. E. Fritzen. 1998. Influences of Hunting on the Behavior of WhiteTailed Deer: Implications for Conservation of the Florida Panther. Conservation Biology, 12 (6):1359-1364.

Kobelkowsky, R. S. 2000. Evaluación del hábitat y estructura de la población de venado cola blanca (Odocoileus virginianus) en la región central de la Sierra Fría, Aguascalientes. Tesis de Maestría. Colegio de Postgraduados. Montecillo, Texcoco, Edo. de México. 87 p.

Leopold, A. S. 1985. Fauna Silvestre de México. Tercera reimpresión. Editorial Pax. México, D. F. $608 \mathrm{p}$.

Mandujano, S., S. Gallina, G. Arceo \& L. A. Pérez-Jiménez. 2004. Variación Estacional del Uso y Preferencia de los Tipos Vegetacionales por el Venado Cola Blanca en un Bosque Tropical de Jalisco. Acta Zoológica Mexicana (n.s.) 20(2): 45-67.

Manly, B., L. McDonald, \& D. Thomas. 1993. Resourse Selection by Animals. Statistical design and analysis for field studies. Chapman \& Hall. London. $177 \mathrm{p}$.

Marshal, J. P., V. C. Bleich, P. R. Krausman, M. L. Reed, \& N. G. Andrew. 2006. Factors Affecting Habitat Use and Distribution of Desert Mule Deer in an Arid Environment. Wildlife Society Bulletin 34 (3): 609-619.

Medina, F. A. 1986. Programa de conservación y aprovechamiento cinegético del venado cola blanca en el estado de Aguascalientes. Pp. 62 - 69. En: Roa R. M. A. (Ed.) Curso de Actualización de la División de Estudios de Postgrado de la facultad de Medicina Veterinaria y Zootécnia de la UNAM. UNAM-FMVZ.

Medina-Torres, S. M. 2006. Delimitación y Diagnostico Preliminar de la Unidad de Manejo Forestal Regional “Asociacion Sierra del Laurel, A.C.”. Calvillo Aguascalientes, México. 60 p.

Moore, W. R., W. L. Myers, W. Gaines, J. Agee, K. Raedeke, \& S. Bushnell. 2003. Winter Habitat Use. 28-52 Pp. In: Myers, W.L. (ed.) Observations of Mule Deer Habitat Use, Movements, and Survival in Chelan. Washington Department of Fish and Wildlife. Olympia, Washington.

Morrison, S. F., G. J. Forbes, S. J. Young, \& S. Lusk. 2003. Within-yard habitat use by white-tailed deer at varying winter severity. Forest Ecology and Management 172:173-182

Mysterud A. \& Ims, R. A. 1998. Functional Responses in Habitat Use: Availability Influences Relative Use in Trade-Off Situations. Ecology 79:1435-1441.

Olson, R. 1992. White-Tailed Deer Habitat Requirements \& Management in Wyoming. Department of Renewable Resources. College of Agriculture. University of Wyoming. 19 p.

Ortiz-Martínez T., S. Gallina, M. Briones-Salas \& G. González. 2005. Densidad Poblacional y Caracterización del Hábitat del Venado Cola Blanca (Odocoileus Virginianus Oaxacensis, Goldman y Kellog, 1940) en un Bosque Templado de la Sierra Norte de Oaxaca, México. Acta Zoológica Mexicana (n.s.) 21(3): 65-78.

Romo, D. M. 1987. Dinámica de la Población del Venado Cola Blanca (Odocoileus. virginianus) en la Sierra de San Blas de Pabellón en el Estado de Aguascalientes, México. Tesis Profesional. Universidad Autónoma de Aguascalientes. Aguascalientes, México. 108 p.

Rzedowski, J. \& R. McVaugh. 1966. La vegetación de Nueva Galicia. Contr. Univ. Mich. Herb. 9: 1123. 
SAS. 2007. SAS Institute Inc., SAS Campus Drive, Cary, North Carolina 27513, USA. En: http://www.colpos.mx/servicios/sas/windowsxp.htm.

SDR. 2007. Manual para el Manejo de Venado Cola Blanca (Odocoileus virginianus mexicanus) en la Mixteca Poblana. Secretaria de Desarrollo Rural del Estado de Puebla. Gobierno del Estado de Puebla. Archivo digital. En: http://www.sdr.gob.mx

Segura, L. W. 1998. Application of the HEP Methodology and Use of GIS to Identify Priority Sites for the Management of White-Tailed Deer. Pp. 127-137 In: Savitsky, G. B. \& Lacher Jr., E. T. (ed.). GIS Methodologies for Developing Conservation Strategies. Tropical Forest Recovery and Wildlife Management in Costa Rica. Columbia University Pres, New Yor, U.S.A.

SEMARNAP. 1997. Programa de Conservación de la Vida Silvestre y Diversificación Productiva en el Sector Rural: 1997 - 2000. Secretaría de Medio Ambiente, Recursos Naturales y Pesca. México, $207 \mathrm{p}$.

SEMARNAT-CONAFOR. 2004. Inventario nacional forestal y de suelos 2004 - 2009. Manual y procedimientos para el muestreo de campo. Archivo PDF. En: http://www.conafor.gob.mx/ portal/home.php

Siqueiros-Delgado M., G. García-Regalado, \& M. De la Cerda-Lemus. 2006. Selva baja caducifolia o matorral subtropical en el estado de Aguascalientes, México. Memoria. Contribuciones presentadas en la modalidad de cartel. Congreso Mexicano de Ecología 2006. p 127.

Uresk, D. W., T. A., Benzon, K. E. Severson, \& L. Benkobi. 1999. Characteristics of White-Tailed Deer Fawn Beds, Black Hills, South Dakota. Great Basin Naturalist 59:348-354.

Villarreal, G. J. G. 1999. Venado Cola Blanca. Manejo y Aprovechamiento Cinegético. Unión Ganadera Regional de Nuevo León. Monterrey, Nuevo León, México. 401 p.

Villarreal Espino-Barros, O. A. \& M. M. Marín-Fuentes. 2005. Agua de origen Vegetal para el Venado Cola Blanca Mexicano. Archivos de Zootecnia 54:191-196.

Villarreal Espino-Barros O. A., V. R. Guevara, G. F. J. Franco, J. C. Castillo Correo, M. I. Cortes, A. L. E. Campos, C. J. C. Rodríguez y V. G. Guevara. 2007. Estimación de la Densidad Poblacional del Venado Cola Blanca, en la Región de la Mixteca Poblana. XXX Congreso Nacional de Buiatría. Archivo digital. En: www.fmvz.unam.mx/bovinotecnia/BtRgorg001.pdf

Villalobos, S. V. 1998. El Venado Cola Blanca en la Sierra Fría de Aguascalientes. Cuadernos de Trabajo Agricultura y Recursos Naturales. No. 89. Gobierno del Estado de Aguascalientes, México. $73 \mathrm{p}$.

Wayne, R. K. 2001. Effect of Scale on Habitat Selection of Female White-Tailed Deer in The Central Black Hills, South Dakota and Wyoming. PhD. thesis. South Dakota State University. 232 p. 